Sädhanā, Vol. 26, Part 5, October 2001, pp. 413-437. (C) Printed in India

\title{
Fundamentally excited flow past a surface-mounted rib. Part II: Kinetic energy budget details
}

\author{
P K PANIGRAHI
}

Department of Mechanical Engineering, Indian Institute of Technology, Kanpur 208 016, India

e-mail: panig@iitk.ac.in

MS received 29 December 1999; revised 19 February 2001

\begin{abstract}
This paper presents the detailed turbulent kinetic energy budget and higher order statistics of flow behind a surface-mounted rib with and without superimposed acoustic excitation. Pattern recognition technique is used to determine the large-scale structure magnitude. It is observed that most of the turbulence contributions after the reattachment region are from the large-scale structures contrary to the belief that mostly random turbulent structures are present after reattachment. The dissipation is not a small-scale phenomena only. It may result due to the interaction between large-scale structures. From the results of higher order moments, the outer edge of the shear layer is observed to be non-Gaussian in nature with significant deviation from the Gaussian skewness and flatness value. The kinetic energy budget results show positive intermodal production in the outer edge of the shear layer indicating the presence of back scattering. The non-Gaussian velocity distribution, ejection motions and back-scattering present in the outer shear layer may be conjectured to be correlated with each other.
\end{abstract}

Keywords. Pattern recognition method; higher order moments; kinetic energy budget; turbulator; coherent structures.

\section{Introduction}

Ribbed duct flows are encountered in a variety of practical applications, for example, turbine blade cooling and heat exchangers, where it is desired to enhance the mixing in the separated shear layer behind the rib. The reattaching shear layer behind a surface-mounted rib is dominated by large-scale structures. It is expected that the flow and heat transport mechanisms would be largely influenced by the dynamics of these structures. Thus, there is a need for identifying the mechanisms associated with the production and transport of coherent turbulent structures. The mixing enhancement in this separated flow can be obtained by manipulating these large-scale structures. Based on studies reported for perturbed shear layers (Ho \& Huere 1984) it is expected that the superimposed excitation

A list of symbols is given at the end of the paper 
on the reattaching shear layer would promote mixing and shear layer growth. However, the selection of proper excitation parameters requires the complete understanding of turbulent transport quantities and their evolution in the reattaching shear layer. Hence, the detailed turbulent statistics and energy budget of the reattaching shear layer behind a surface-mounted rib is the focus of this paper.

The influence of shear-layer excitation on the development and evolution of coherent structures behind a backward-facing step has been investigated by several investigators (Bhattacharjee et al 1986; Roos \& Kagelman 1986; Hasan 1992). Roos \& Kagelman (1986) observed sufficient reduction in the reattachment length when forced at the non-dimensional frequency (based on freestream velocity and step height) greater than 0.25 , due to the completion of at least one stage of pairing prior to the reattachment. Bhattacharjee et al (1986) observed that the spreading rate can be increased most effectively by forcing at a non-dimensional frequency (based on step height and free stream velocity) between 0.2 and 0.4. Hasan (1992) observed two distinct modes of instability: the shear layer mode $\left(\mathrm{St}_{\theta} \simeq 0.012\right)$ and the step mode $\left(\mathrm{St}_{h} \simeq 0.185\right)$. All these investigators (Bhattacharjee et al 1986; Roos \& Kagelman 1986; Hasan 1992) reported time-mean flow measurements to show the effect of excitation on the reattaching shear layer. However, measurements reporting the detail turbulent structures and how they are influenced by forcing have not been reported. The ribbed duct flow represents a reattaching shear layer different from that past a backward-facing step due to flow separation upstream of the rib. The effect of single frequency excitation on the reattaching shear layer past a surface-mounted rib has not yet been investigated. This investigation attempts to explain the effect of excitation on the turbulent transport quantities of a reattaching shear layer past a surfacemounted rib.

\section{Turbulent kinetic energy budget}

The kinetic energy equations of turbulent flow helps to explain the production, transport and dissipation of velocity and pressure fluctuations. Thus, an overview of the kinetic energy equations of the mean flow and fluctuations will be discussed here and the results for the ribbed duct flow will be presented in the results and discussions section. The conventional turbulent energy budget can be expressed by the the following equation (Kundu 1990).

$$
\frac{D}{D t}\left(\overline{\frac{1}{2} u_{i}^{\prime 2}}\right)=-\frac{\delta}{\delta x_{j}}\left(\frac{1}{\rho_{o}} \overline{p u_{j}}+\frac{1}{2} \overline{u_{i}^{\prime 2} u_{j}^{\prime}}-2 v \overline{u_{i}^{\prime} e_{i j}}\right)-\overline{u_{i}^{\prime} u_{j}^{\prime}} U_{i, j}-2 v \overline{e_{i j} e_{i j}},
$$

where

$$
e_{i j}=1 / 2\left(u_{i, j}^{\prime}+u_{j, i}^{\prime}\right)=\frac{1}{2}\left(\frac{\delta u_{i}^{\prime}}{\delta x_{j}}+\frac{\delta u_{j}^{\prime}}{\delta x_{i}}\right) .
$$

The left hand side of the above equation is the convection of turbulent kinetic energy representing the transport of turbulent energy by the mean motion and can be simplified for a 2-D flow as the convection term:

$$
\text { convection }=U_{j} \frac{\delta}{\delta x_{j}}\left(\frac{1}{2} \overline{u_{i}^{\prime} u_{i}^{\prime}}\right)=U \frac{\delta q^{2}}{\delta x}+V \frac{\delta q^{2}}{\delta y},
$$

where,

$$
q^{2}=1 / 2\left(u^{\prime 2}+v^{\prime 2}+w^{\prime 2}\right)
$$


By assuming that $w^{\prime 2}$ lies half way between $u^{\prime 2}$ and $v^{\prime 2}$ (Driver \& Seegmiller 1982; Chandrasuda \& Bradshaw 1981) we have:

$$
q^{2}=3 / 4\left(u^{2}+v^{2}\right) .
$$

The first term on the RHS side of the above equation represents the transport of turbulence by pressure fluctuations and is obtained here as a balance of other terms. The second term on the RHS represents the diffusion due to velocity fluctuation. Assuming $\overline{v^{\prime} w^{\prime 2}}=\overline{v^{\prime 3}}$ and $\overline{u^{\prime} w^{\prime 2}}=\overline{u^{\prime} v^{\prime 2}}$ (Zhou et al 1996), the second term can be simplified for 2-D flow as:

$$
\begin{aligned}
\text { diffusion }=-\frac{1}{2} \frac{\delta}{\delta x_{j}}\left(\overline{u_{i}^{\prime 2} u_{j}^{\prime}}\right) & =-\frac{\delta}{\delta x}\left(\overline{u^{\prime} q^{2}}\right)-\frac{\delta}{\delta y}\left(\overline{q^{2} v^{\prime}}\right) \\
& =-\frac{1}{2} \frac{\delta}{\delta x}\left(\overline{u^{\prime 3}}\right)-\frac{\delta}{\delta x}\left(\overline{u^{\prime} v^{\prime 2}}\right)-\frac{1}{2} \frac{\delta}{\delta y}\left(\overline{u^{\prime 2} v^{\prime}}\right)-\frac{\delta}{\delta y} \overline{v^{\prime 3}} .
\end{aligned}
$$

Alternatively the assumption of Driver \& Seegmiller (1982) and Chandrasuda \& Bradshaw (1981), that $w^{\prime 2}$ lies midway between $u^{\prime 2}$ and $v^{\prime 2}$, can be used and the diffusion can then be calculated using the following equation:

$$
\text { diffusion }=-\frac{\delta}{\delta x}\left(\frac{3}{4}\left(\overline{u^{\prime 3}}+\overline{u^{\prime} v^{\prime 2}}\right)\right)-\frac{\delta}{\delta y}\left(\frac{3}{4}\left(\overline{v^{\prime 3}}+\overline{v^{\prime} u^{\prime 2}}\right)\right) .
$$

The calculation of the diffusion contribution using both the above approximations is presented in figure 1. Both assumptions give comparable predictions. This indicates the appropriateness of either assumption for the diffusion calculation. Subsequently, the assumption used by Driver et al (1982) and Chandrasuda et al (1981) is used here for the calculation of diffusion.

The third term on the RHS of (1) is the viscous diffusion term and usually is neglected at high Reynolds number. The fourth term represents the production of turbulent kinetic energy, which for a two-dimensional flow can be simplified as:

$$
\text { production }=-\overline{u_{i} u_{j}} U_{i, j}=-\overline{u^{\prime 2}} \frac{\delta U}{\delta x}-\overline{u^{\prime} v^{\prime}} \frac{\delta U}{\delta y}-\overline{v^{\prime 2}} \frac{\delta U}{\delta y}-\overline{u^{\prime} v^{\prime}} \frac{\delta V}{\delta x} .
$$

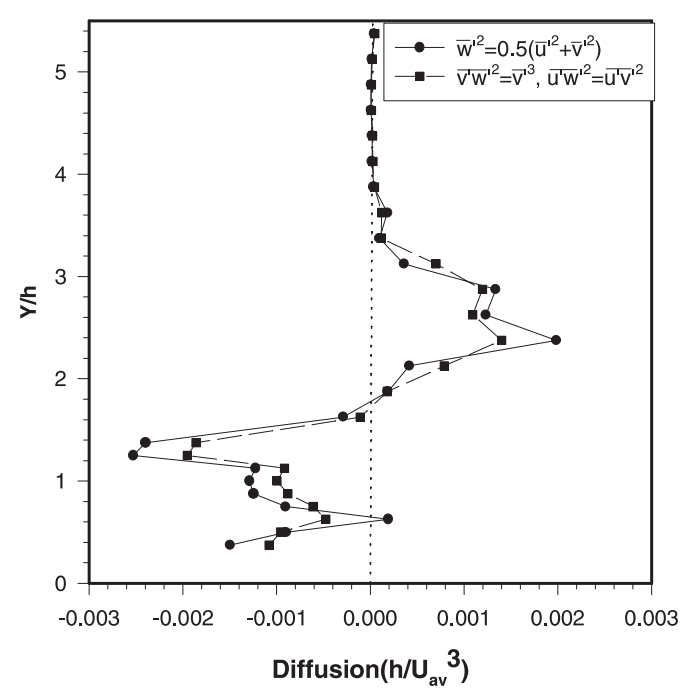

Figure 1. Verification of assumptions in diffusion calculation for the excited flow. 
By using continuity equation, the production term can be written as:

$$
\text { production }=-\left(\overline{u^{\prime 2}}-\overline{v^{\prime 2}}\right) \frac{\delta U}{\delta x}-\overline{u^{\prime} v^{\prime}}\left(\frac{\delta U}{\delta y}+\frac{\delta V}{\delta x}\right)
$$

where, the first term is production due to normal stress and the second term is the production due to shear stress.

The fifth term on the RHS of (1) is the viscous dissipation term. The average rate of dissipation can be obtained by assuming the validity of the postulate of local isotropy: i.e. at sufficiently high Reynolds number, the small-scale turbulent structure is isotropic even when the large-scale structure is not and the streamwise derivatives of the velocity components are related by (Champagne 1978):

$$
\overline{\left(\delta u^{\prime} / \delta x\right)^{2}}=(1 / 2) \overline{\left(\delta v^{\prime} / \delta x\right)^{2}}=(1 / 2) \overline{\left(\delta w^{\prime} / \delta x\right)^{2}} .
$$

Then, the average rate of energy dissipation $(\epsilon)$ can be simplified as (Champagne 1978):

$$
\epsilon=15 v \overline{\left(\delta u^{\prime} / \delta x\right)^{2}} \text {. }
$$

The above velocity fluctuation gradient can be derived from the temporal derivative by using Taylor's approximation:

$$
\frac{\delta}{\delta t}=\bar{U} \frac{\delta}{\delta x} \text {. }
$$

A correction for the dissipation calculation in the presence of high turbulence intensity as suggested by Champagne (1978) is used here as,

$$
\epsilon_{m}=\epsilon\left(1+\frac{\overline{u^{\prime 2}}}{\overline{U^{2}}}+2 \times \frac{\overline{v^{\prime 2}}+\overline{w^{\prime 2}}}{\overline{U^{2}}}\right),
$$

where, $\epsilon_{m}$ denotes the value of dissipation rate inferred from the measured temporal derivative obtained using Taylor's approximation.

\subsection{Kinetic energy of coherent eddies}

The kinetic energy budget from the coherent eddies is obtained here by using the formulation suggested by Hussain (1970) as:

$$
\begin{gathered}
\left.U_{j} \frac{\delta}{\delta x_{j}}\left(\frac{1}{2} \overline{u_{c i} u_{c i}}\right)=-\frac{\delta}{\delta x_{j}} \overline{\left(u_{c j}\left(p_{c}+\frac{1}{2} u_{c i} u_{c i}\right)\right.}\right)+\left(-\overline{u_{c i} u_{c j}}\right) \frac{\delta U_{i}}{\delta x_{j}}-\overline{\left(\left\langle u_{r i} u_{r j}\right\rangle\right) \frac{\delta u_{c i}}{\delta x_{j}}} \\
-\frac{\delta}{\delta x_{j}} \overline{\left(u_{c i}\left\langle u_{r i} u_{r j}\right\rangle\right)}+v \frac{\delta}{\delta x_{j}} \overline{\left(u_{c i}\left(\frac{\delta u_{c i}}{\delta x_{j}}+\frac{\delta u_{c j}}{\delta x_{i}}\right)\right)}-\frac{v}{2} \overline{\left.\left(\frac{\delta u_{c i}}{\delta x_{j}}+\frac{\delta u_{c j}}{\delta x_{i}}\right)^{2}\right)} .
\end{gathered}
$$

The left hand side of the above equation represents the coherent convection, and using an assumptions similar to that made in the earlier section, the coherent convection term can be simplified for 2-D flow as:

$$
\text { coherent convection }=V \frac{\delta}{\delta y}\left(\overline{q_{c}^{2}}\right)+U \frac{\delta}{\delta x}\left(\overline{q_{c}^{2}}\right),
$$

where $q_{c}^{2}$ is the coherent turbulent kinetic energy equal to $(3 / 4)\left(u_{c}^{2}+v_{c}^{2}\right)$. 
The first term on the RHS of the energy budget equation, (7), is the coherent pressure diffusion, which is assumed here to be equal to the pressure diffusion value obtained from the conventional double decomposition method. This assumption is based on earlier observations which estimated the coherent velocity component to be the significant part (see figures 2 and 3). It is therefore assumed that the coherent pressure fluctuation is also a significant part of the total pressure fluctuation.

The second and third terms on the RHS of (7) are the coherent diffusion and coherent production respectively and are simplified as:

$$
\begin{aligned}
& \text { coherent diffusion }=-\frac{\delta}{\delta x}\left(\frac{3}{4}\left(\overline{u_{c}^{3}}+\overline{u_{c} v_{c}^{2}}\right)\right)-\frac{\delta}{\delta y}\left(\frac{3}{4}\left(\overline{v_{c}^{3}}+\overline{v_{c} u_{c}^{2}}\right)\right), \\
& \text { coherent production }=-\left(\overline{u_{c}^{2}}-\overline{v_{c}^{2}}\right) \frac{\delta U}{\delta x}-\overline{u_{c} v_{c}}\left(\frac{\delta U}{\delta y}+\frac{\delta V}{\delta x}\right) .
\end{aligned}
$$

The fifth term on the RHS of (7), known as the diffusion of coherent energy by random velocity fluctuation, is assumed here to be negligible. The sixth term is viscous diffusion and can be assumed to be negligible at high Reynolds number. The last term is the coherent viscous dissipation, which is calculated using Taylor's hypothesis, similar to the conventional double decomposition method. The fourth term known as the intermodal production terms between the random and the coherent contributions is calculated by a balance of the calculated terms in (7).

\section{Results and discussion}

The experimental set-up used for this work was explained by the author in his earlier communication (Panigrahi 2001). The pattern recognition technique for evaluation of large-scale structure magnitude used in this paper was also discussed in detail in the above communication. The mean flow measurements, Reynolds stresses, higher order moments, the turbulent kinetic energy budget and the coherent energy budget of the flow behind the surface-mounted rib are explained in the following sections.

\subsection{Mean flow measurements}

The evolution of the mean streamwise velocity and cross stream velocity before and after reattachment, for both the excited and the unexcited case are presented in figure 4 . The $u$-velocity is normalized by the maximum velocity at the corresponding $X / h$ locations, while the $v$-velocity is normalized with the average $u$-velocity across the channel. The effect of forcing on the mean $v$-velocity is observed to be considerably more pronounced than on the $u$-velocity and manifests itself over the entire shear layer region. This reflects the greater receptivity of the $v$-momentum to the imposed perturbation. At $X / h=1.5$, the $v$-velocity is positive in the upper half of the separated shear layer for the unexcited case indicating the effect of the upward deflection of the shear layer, while for the excited case, the $v$-velocity is mostly negative at this location indicating the downward motion of the flow. This earlier transition to downward motion for the excited case is a precursor for early reattachment. It should be noted that the reattachment length for the unexcited case was observed to be equal to $5.5 \mathrm{~h}$ and for the excited case was equal to $4.0 h$ supporting the above observations. At $X / h=9.0$, the maximum absolute $v$-velocity value for the unexcited case is $41.66 \%$ more than the excited case. The 

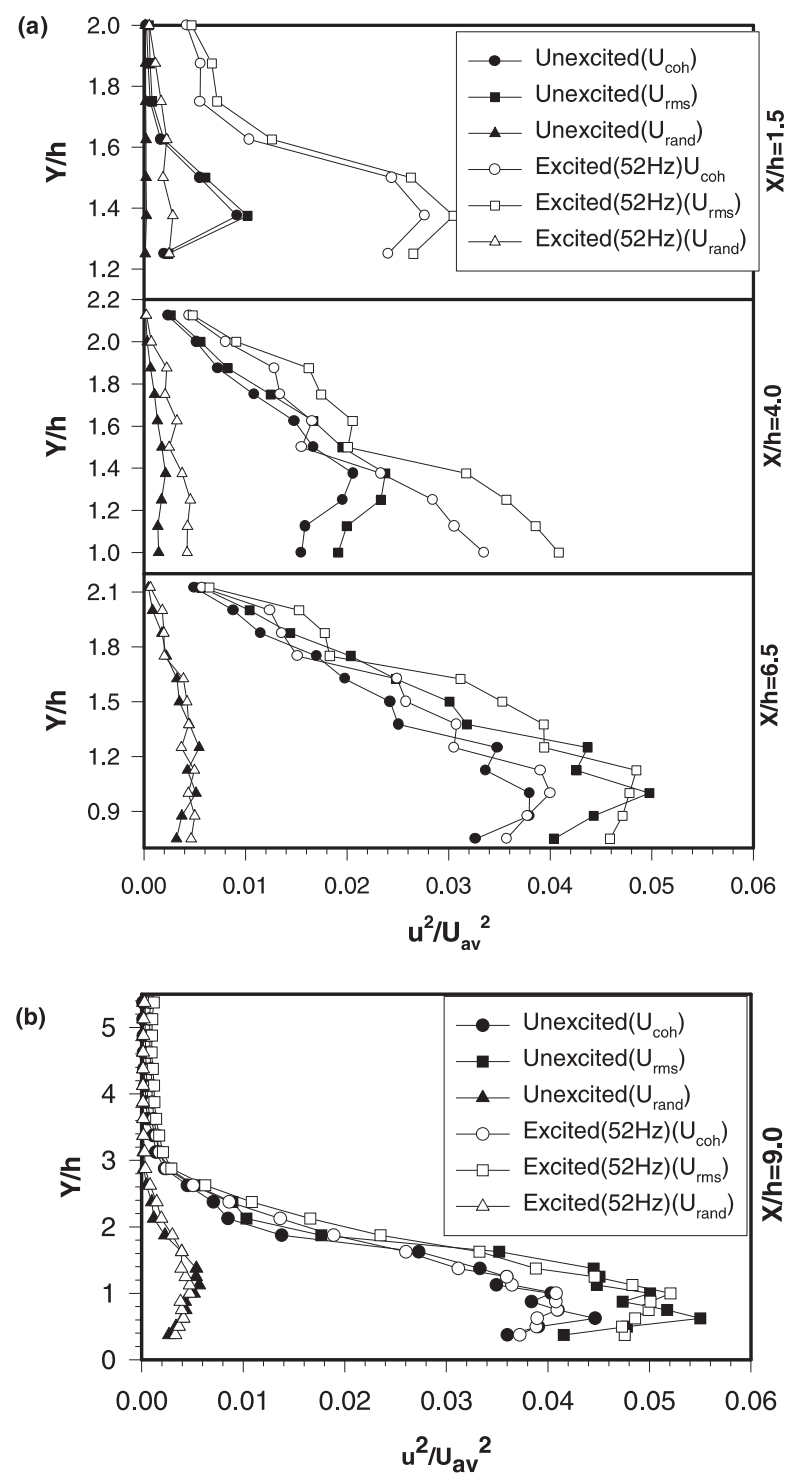

Figure 2. Streamwise fluctuation profile at (a) $X / h=1.5,4.0$, and 6.5 (near and upstream of reattachment location) and (b) at $X / h=9.0$ (downstream of reattachment) for both excited and unexcited cases.

smaller $v$-velocities for the excited cases imply that the flow recovery has proceeded to a greater extent. This, in turn, confirms that flow reattachment occurs earlier for the excited case.

At $X / h=1.5$, the effect of excitation on the mean $u$-velocity can be seen primarily up to a $Y / h$ value of 1.5 . For $Y / h>1.7$ the $u$-velocity gradient are small, and no effect of excitation is observed due to the absence of velocity gradient. In the vicinity of reattachment $(X / h=6.5)$ and beyond reattachment $(X / h=9.0)$ the effect of excitation is limited to the near wall region only. The mean $u$-velocity close to the wall is observed to be larger with excitation than that for the unexcited case indicating larger momentum transport to the near-wall boundary layer region due to the imposed excitation. 


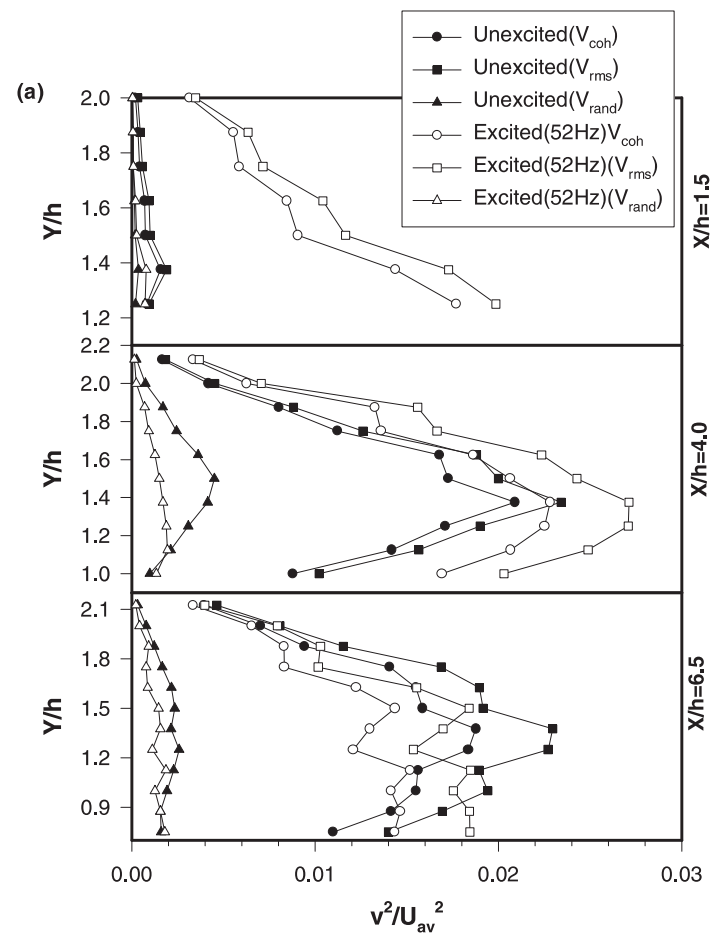

(b)

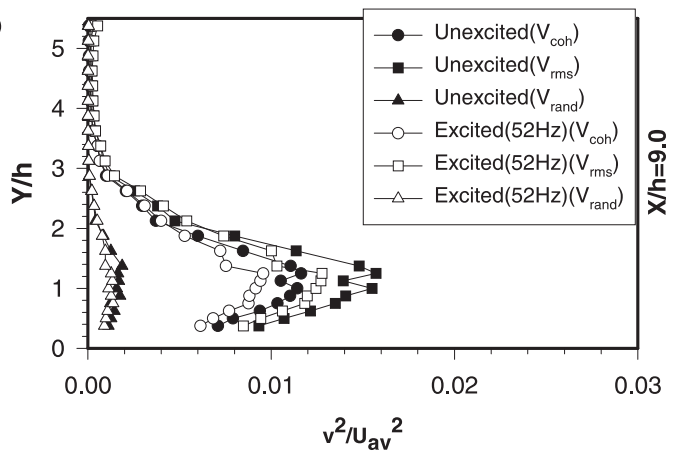

Figure 3. Cross-stream fluctuation profile at (a) $X / h=1.5,4.0$, and 6.5 (near and upstream of reattachment location) and (b) at $X / h=9.0$ (downstream of reattachment) for both excited and unexcited cases.

\subsection{Reynolds stresses}

The velocity fluctuations (streamwise and cross-stream) and shear stresses for both the excited and unexcited flow are presented in figures 2,3 and 5 respectively at $X / h=1.5$, 4.0, 6.5 and 9.0. The values are normalized by the square of the average velocity in the channel to assess the effect of excitation on the magnitude of the fluctuations and their distributions at different $X / h$ and $Y / h$ locations. In the near field region $(X / h=1.5$ and 4.0$)$, the streamwise and cross-stream fluctuations for the excited case are considerably larger than those for the unexcited case. However, the differences diminish in the downstream direction, and a cross-over occurs in the vicinity of reattachment. At $X / h=6.5$, the streamwise fluctuations for the unexcited and excited cases are comparable, while the cross-stream fluctuations for the excited cases are less than that of the unexcited 


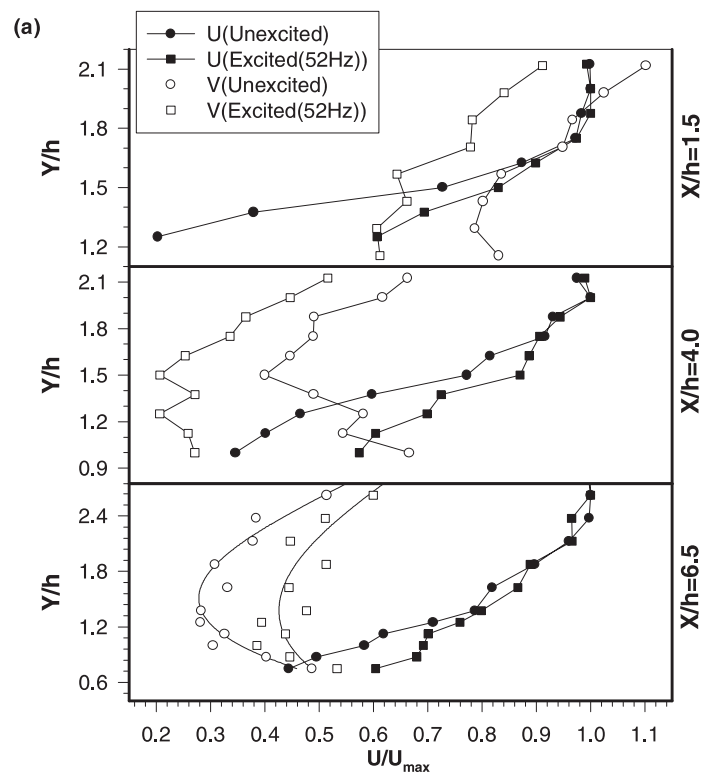

$\begin{array}{lllllll}-0.14-0.12-0.10-0.08 & -0.06-0.04-0.02 & 0.00 & 0.02 & 0.04 & 0.06\end{array}$

$\mathrm{V} / \mathrm{U}_{\mathrm{av}}$

(b)

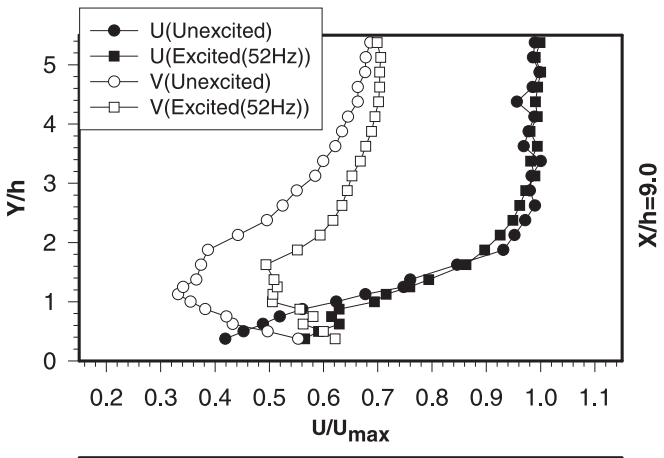

$-0.14-0.12-0.10-0.08-0.06-0.04-0.020 .000 .020 .040 .06$

$\mathrm{V} / \mathrm{U}_{\mathrm{av}}$
Figure 4. Mean velocity profiles at (a) $X / h=1.5,4.0$ and 6.5 (near and upstream of reattachment location) and (b) at $X / h$ $=9.0$ (downstream of reattachment) for both excited and unexcited cases.

cases. In the far field region $(X / h=9.0)$, the velocity fluctuations (streamwise and cross-stream) for the excited case are smaller than those of the unexcited case. This is due to the earlier development and saturation of energy of the excited shear layer compared to the unexcited case. For a backward-facing step, Hasan (1992) also observed lower streamwise turbulence intensity around the reattachment region for the excited case compared to that for the unexcited case.

The contribution from the coherent part of the fluctuation, calculated from using the pattern recognition method are also shown in figures 2 and 3. In both before and after reattachment region, the coherent components are significantly larger than the random component which indicates that the large-scale eddies dominate the development of the reattaching shear layer. It may also be observed that the imposed oscillation mostly affects the coherent velocity fluctuations, while the random part is not noticeably affected. 


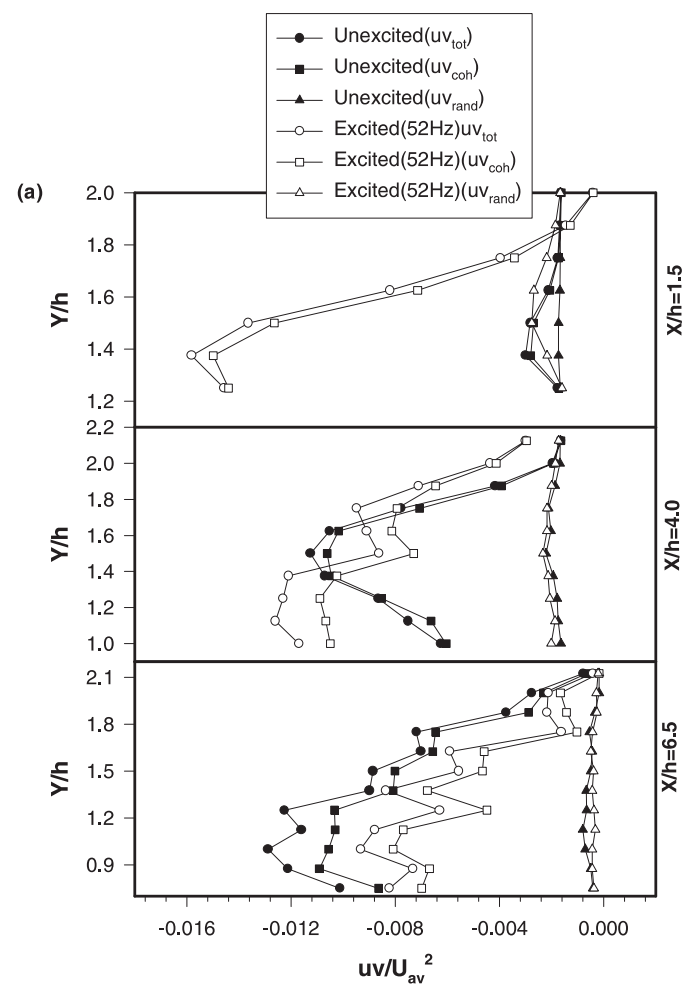

(b)

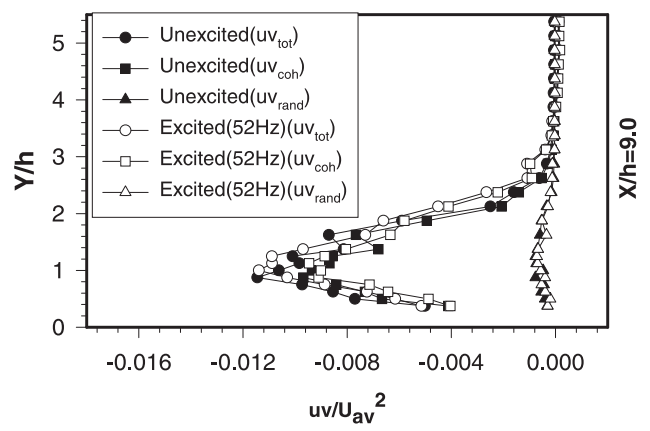

$\frac{11}{x}$

II

Figure 5. Shear stress profile at (a) $X / h$ $=1.5,4.0$, and 6.5 (near and upstream of reattachment location) and (b) at $X / h=9.0$ (downstream of reattachment) for both excited and unexcited cases.

At $X / h=1.5$ and 4.0 both the coherent and the random stream-wise velocity fluctuations for the excited case are larger than that of the unexcited case. At $X / h=6.5$ and 9.0, the random fluctuations and the coherent fluctuations of the stream-wise velocity are comparable to each other for both excited and unexcited cases. Thus, the random and coherent component of velocity fluctuations are correlated with each other. Overall, most of the contributions to the fluctuations are from the coherent part both in the near-field as well as in the far-field region.

In the vicinity of reattachment $(X / h=6.5)$ and beyond $(X / h=9.0)$, the peak in the $v$-velocity fluctuation (see figure 3 ) can be observed to occur further away from the wall compared to the $u$-fluctuation (see figure 2). This is due to the strong inhibiting effect of the solid surface on the $v$-fluctuation compared to the $u$-fluctuation. Zhou et al (1996), using no slip conditions and continuity equations argued that at 
the wall, both the $v$-fluctuation and the gradient of $v$-fluctuation are zero and therefore the influence of the wall is more pronounced on the $v$-fluctuation close to the wall compared to the $u$-fluctuation. The present study corroborates this argument, and due to this wall-damping effect, the peak in the $v$-fluctuation profile occurs at a higher $Y / h$ value.

The evolution of the shear stresses (coherent, total and random) at $X / h=1.5,4.0,6.5$ and 9.0 is presented in figure 5. Similar to the velocity fluctuations and mean velocities, the effect of excitation can be observed to be more pronounced in the near-field region. At $X / h=6.5$, the shear stress for the excited case is observed to be smaller than the unexcited case. Thus, the turbulent shear stress in the vicinity of reattachment is less for the excited flow. As the flow recovers downstream of reattachment, the shear stresses for the two cases become comparable (at $X / h=9.0$ ). The excitation induced reduction in shear stress between reattachment and $X / h=9.0$, combined with reduction in the reattachment length, points to the potential of using excitation to enhance heat transfer (due to smaller recirculation zone) with no drag penalty.

At $X / h=6.5$ and 9.0, two peaks can be observed in the turbulent intensity and shear stress profiles (see figures 2-5). One peak is near the wall $(Y / h<1)$ and the second peak is at $Y / h$ near 1.5. This may indicate the presence of two different shear layers, one is the wall boundary layer and the other is the shear layer developing from the separation point, i.e. the edge of the rib. Both the shear layers may be assumed to consist of separate large eddy structures and thus two separate peaks are seen.

The $u$ and $v$ fluctuations normalized with respect to their maximum values are presented in figure 6 for various $X / h$ locations downstream of reattachment. The normalized turbulent profiles collapse reasonably well on to a single asymptotic distribution when normalizing with respect to maximum $u_{r m s}$ and $v_{r m s}$ (see figure 6). At $X / h=9.0$, the profile for the unexcited case shows the maximum deviation and implies that turbulent similarity may not have been established for this case.

The Reynolds stress correlation $\left(\overline{u^{\prime} v^{\prime}} /\left(u_{r m s} v_{r m s}\right)\right)$ for both the unexcited and the excited flow at $X / h=8.5$ and 11.5 are presented in figure 7. The Reynolds stress correlation is a measure of the extent of correlation between the $u$ and $v$ fluctuations. The maximum value of Reynolds stress correlation at $X / h=8.5$ for both the excited and unexcited flow is about 0.46 . The maximum correlation value is observed to be in the range $1.0<Y / h<2.4$. The maximum shear stress occurs at $Y / h \simeq 1.4$ (see figure 5). Hence, there is efficient momentum transport across larger portion of the shear layer. This may be due to the predominant ejection motion present in the outer edge of the shear layer. The maximum value of the Reynolds stress correlation at $X / h=11.5$ is about 0.38 for the unexcited case and 0.32 for the excited case. For the unexcited flow behind the backward-facing step, Chandrsuda \& Bradshaw (1981) observed the shear layer correlation to be equal to 0.47 near the reattachment region which subsequently falls to about 0.4 after reattachment, with no sign of recovery to the usual boundary layer value of 0.45 to 0.5 . A similar drop in the correlation value after reattachment is observed for the present flow with the exception that the drop in the correlation value for the excited case is larger than the unexcited case. For the excited case, the $u$-velocity profile develops faster and is therefore associated with a larger drop in correlation value. The correlation value never recovers to that of a flat plate boundary layer. The turbulent structure of the reattaching shear layer is therefore different from a traditional flat plate boundary layer. 

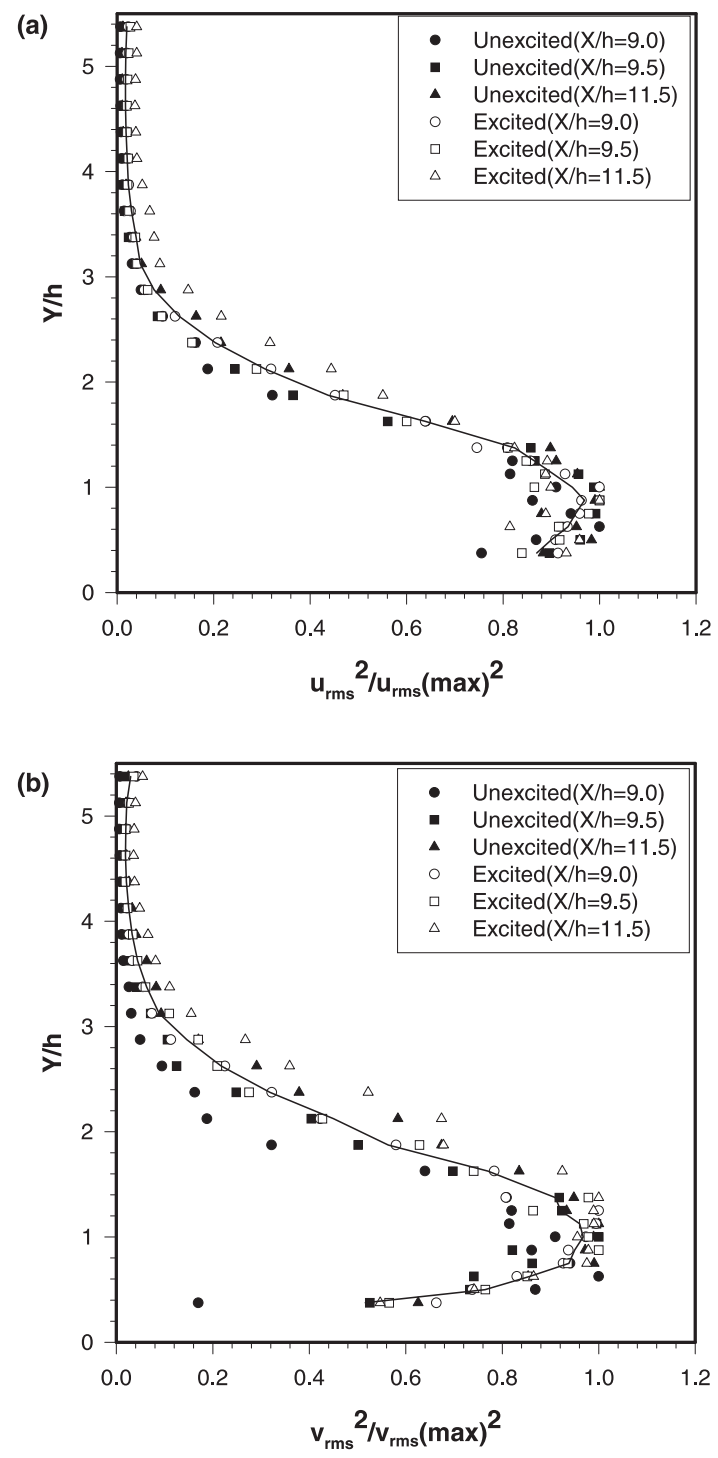

Figure 6. Normalized streamwise (a) and cross-stream (b) fluctuations at various $X / h$ locations for both excited and unexcited cases.

\subsection{Higher order moments}

The higher order correlations, i.e. skewness $\left(\overline{u^{\prime 3}}\right) / \overline{\left(u^{\prime 2}\right)^{3 / 2}}$ and flatness $\left(\overline{u^{\prime 4}} /\left(\overline{u^{\prime 2}}\right)^{2}\right.$, of the streamwise fluctuation of velocity are presented here for both excited and unexcited flow in figures 8 and 9. It was pointed out by Lumley (1981) that much of the information missing from the conventional statistical descriptions may be found in the third and fourth moments.

Outside the shear layer, the skewness of the Gaussian distribution is close to zero (see figure 8), indicating the symmetry of the fluctuation about the local mean velocity (property of a Gaussian distribution). The skewness values for both the excited and unexcited cases are observed to have the highest absolute values in the upper part of the 


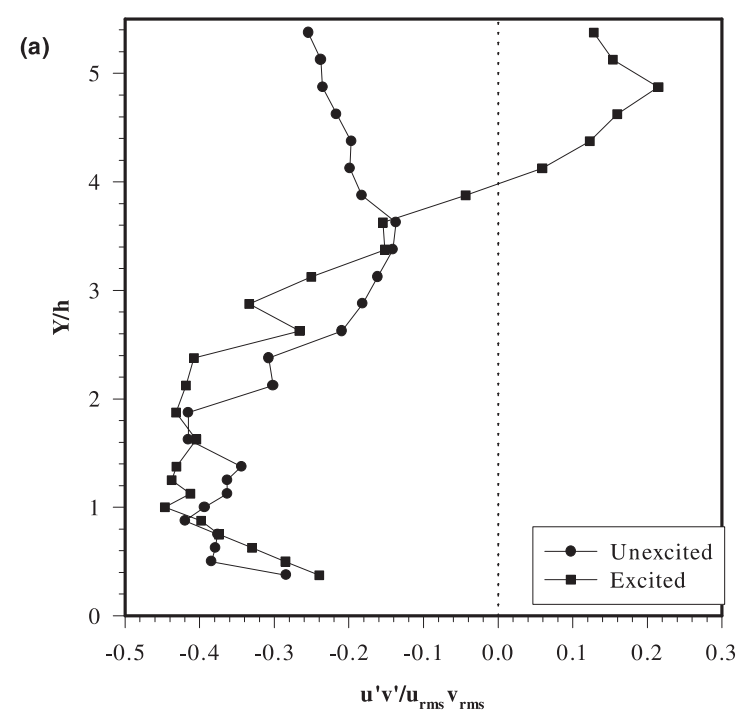

(b)

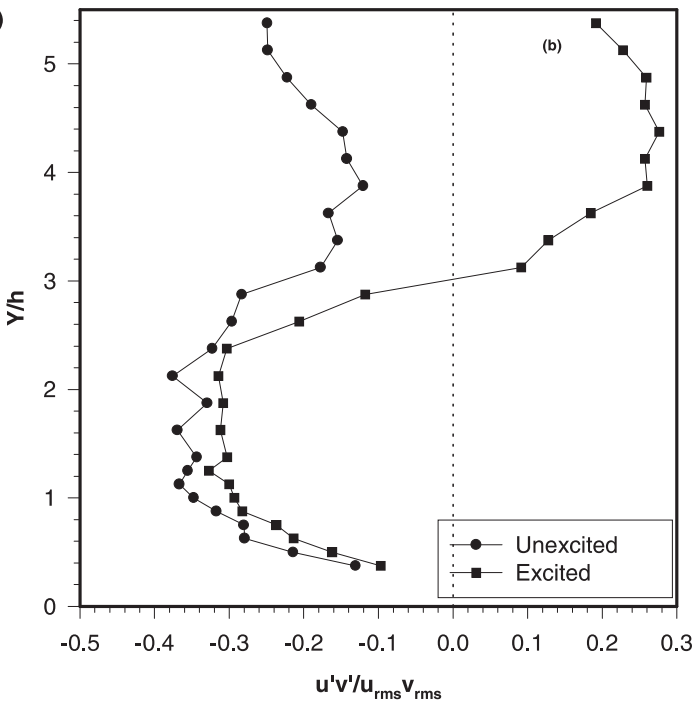

Figure 7. Reynolds stress correlation for both excited and unexcited flows at $X / h$ $=8.5$ (a) and $X / h=11.5(\mathbf{b})$.

shear layer indicating deviation from the Gaussian profile. The maximum absolute value of skewness is higher for the excited case and occurs at a higher $Y / h$ location. The $Y / h$ location that corresponds to the maximum skewness increases in the downstream direction and is due to the increase in the boundary layer growth (see figure 4).

For both the excited and unexcited case, the flatness is observed to have the maximum offset from the Gaussian value of 3.0 at the same $Y / h$ location where the skewness attains maximum values (see figures 8 and 9). The high value of flatness may indicate the presence of high value of flow intermittency (Hinze 1975) with the asymmetry in the negative streamwise $(X)$ direction (negative skewness). Comparing figures 2, 8 and 9 it can be seen that the highest value of coherent and rms fluctuations occur at the $Y / h$ location where the skewness and flatness value are about 0 and 3 respectively. This indicates 
(a)

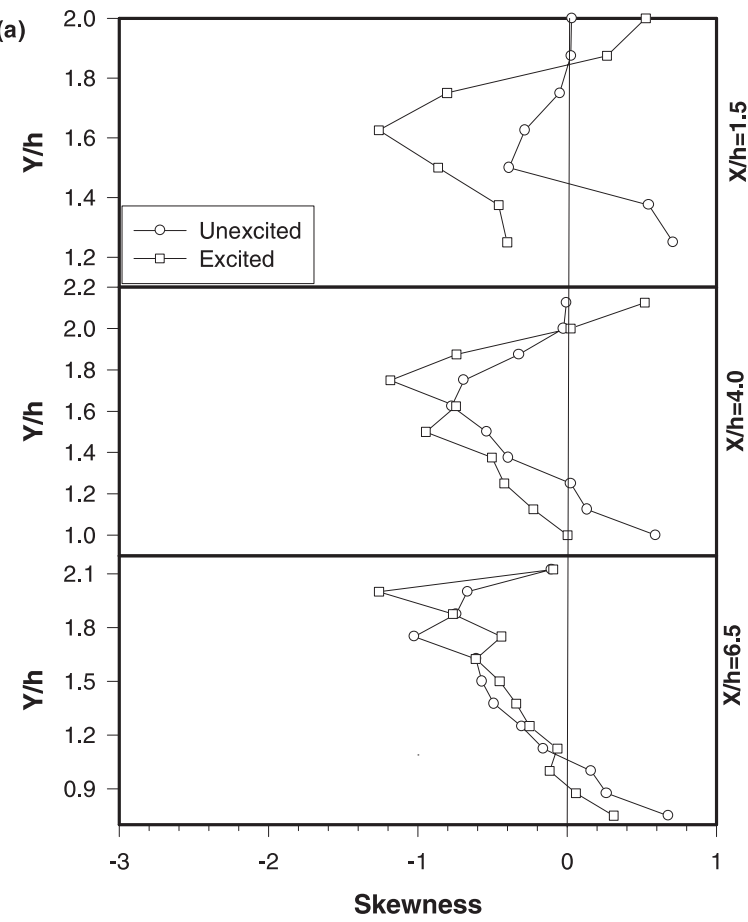

(b)

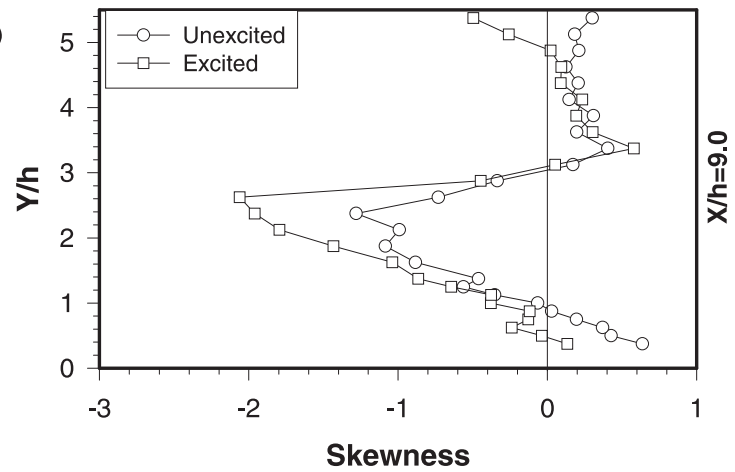

Figure 8. The skewness of $u$ fluctuation at (a) $X / h=1.5,4.0$, and 6.5 (near and upstream of reattachment location) and (b) at $X / h=9.0$ (downstream of reattachment) for both excited and unexcited cases.

that the flow structures in the high turbulent intensity region have Gaussian distribution. Thus, the turbulent structures in the high velocity gradient region do not contribute to the deviation from the Gaussian behaviour. Rather, the high value of intermittency at the edge of the boundary layer due to low frequency fluctuation is the main cause for the deviation from the Gaussian value. To further verify this, we plot the skewness and flatness from the coherent part of the velocity signal obtained using the pattern recognition technique in figure 10. The highest offset in the skewness and flatness value is observed to occur at the same $Y / h$ location for the coherent velocity signal as for the total velocity signal. The difference in the skewness and flatness values between the total and the coherent components is negligible. This implies that the intermittent velocity fluctuation along the edge of the shear layer is mostly from the low frequency large-scale fluctuations and not from small-scale eddies. 
(a)

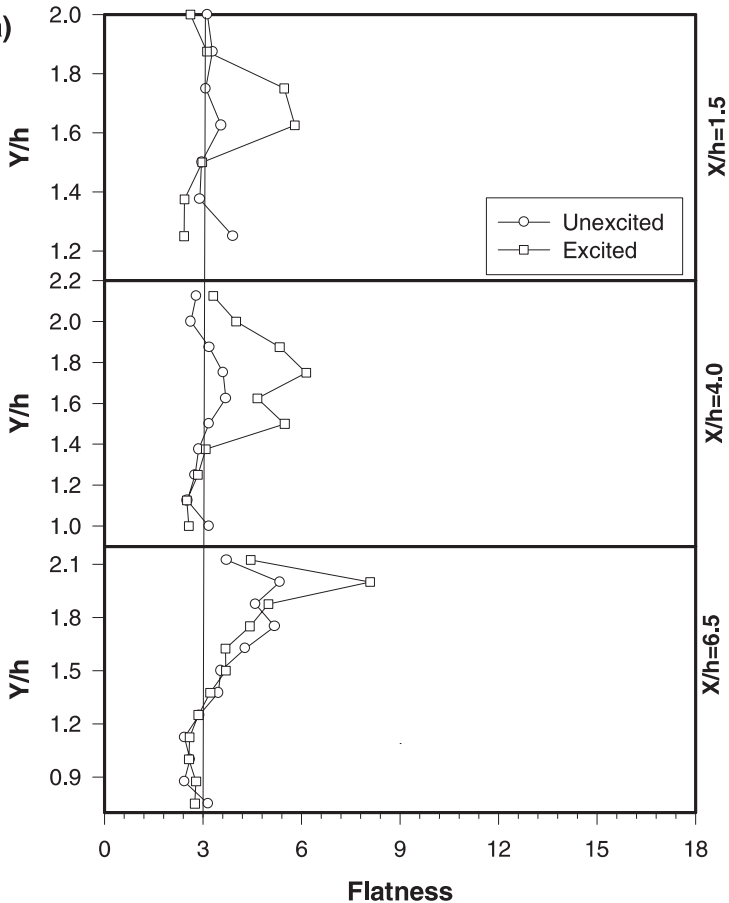

(b)

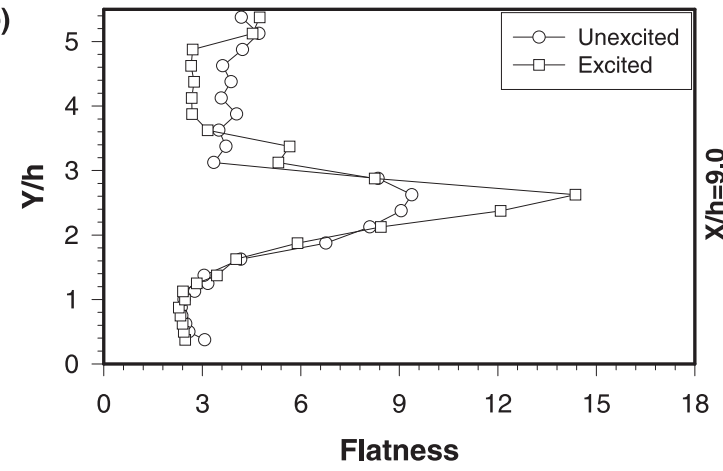

$\frac{7}{x}$

II

Figure 9. The flatness of $u$-fluctuation at (a) $X / h=1.5,4.0$, and 6.5 (near and upstream of reattachment location) and (b) at $X / h=9.0$ (downstream of reattachment) for both excited and unexcited cases.

Comparing figures 8 and 9 with figures 2 and 3, it may be seen that the $Y / h$ locations having non-Gaussian distribution (higher value of skewness and flatness) correspond to the upper part of the shear layer, i.e. the region from maximum turbulence intensity to the edge of the shear layer. It should be mentioned that earlier (Panigrahi 2001), significant ejection motion was observed in the outer shear layer region. In the upper part of the shear layer, entrainment takes place in the form of large-scale engulfment of non-vortical fluid near the interface. These intermittent large-scale entrainment motions may be the primary cause for higher values of skewness and flatness at these locations. To determine if there is a correlation between the ejection motion and higher order moments, the $Y / h$ locations corresponding to the maximum values of skewness and flatness and maximum average hole size of the ejection motion for both excited and unexcited cases are presented in table 1. It may be seen from table 1 that the $Y / h$ locations are almost 
(a)

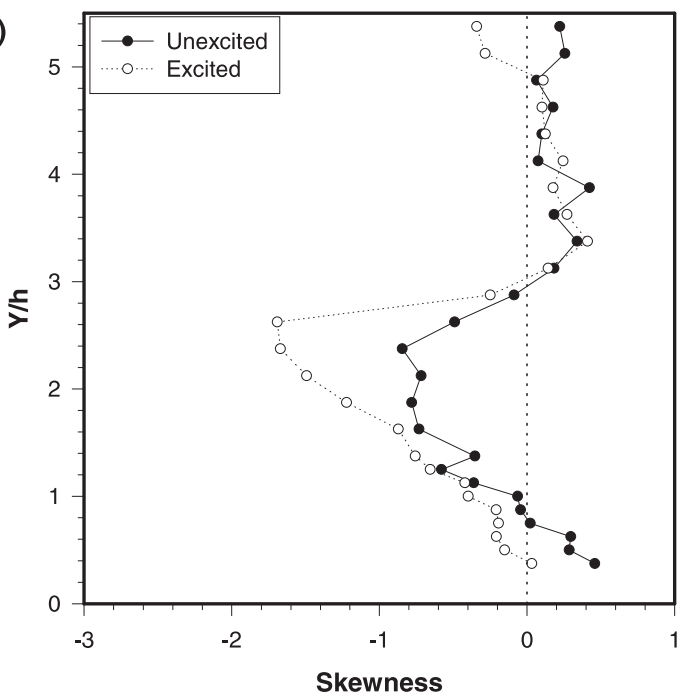

(b)

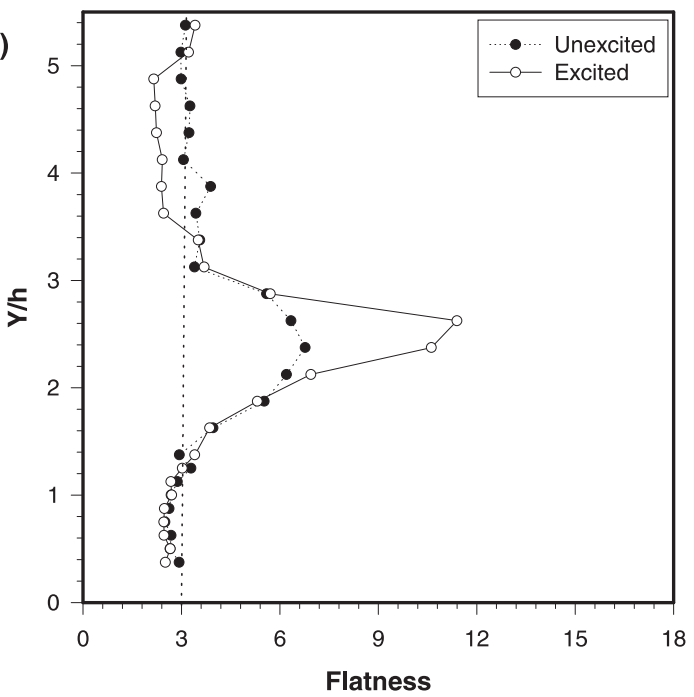

Figure 10. Skewness (a) and flatness (b) profiles of the coherent $u$-velocity for both unexcited and excited cases at various $X / h$ locations.

similar indicating that the higher order moments and ejection motion are correlated. Thus, the large-scale ejection motion primarily contribute to the non-Gaussian nature of the flow in the outer region of the shear layer.

\subsection{Turbulent kinetic energy budget}

In the following paragraphs, the turbulent energy budget of the reattaching shear layer behind the surface-mounted rib is presented and comparison is made with the results of the backward-facing step. The turbulent production profiles for the unexcited and excited case at $X / h=9.0$ are presented in figure 11. Normal stress production can be observed to have its maximum value close to the wall. Normal stress production magnitude is higher than shear stress production magnitude close to the wall resulting in 
Table 1. Comparison of $Y / h$ locations for maximum skewness and flatness value with that for maximum average hole size of ejection motion.

\begin{tabular}{cccccc}
\hline \multirow{2}{*}{$X / h$} & \multicolumn{2}{l}{$\begin{array}{c}Y / h \text { at maximum } \\
\text { skewness } \begin{array}{c}Y \text { and flatness } \\
\text { value }\end{array}\end{array}$} & & \multicolumn{2}{c}{$\begin{array}{c}Y / h \text { at maximum } \\
\text { ejection } \\
\text { average hole } \\
\text { size value }\end{array}$} \\
\cline { 2 - 3 } \cline { 5 - 6 } & Unexcited case & Excited case & & Unexcited case & Excited case \\
\hline 1.5 & 1.55 & 1.6 & & 1.4 & 1.5 \\
4.0 & 1.6 & 1.7 & & 1.6 & 1.6 \\
9.0 & 2.4 & 2.6 & & 1.8 & 2.2 \\
\hline
\end{tabular}

negative total production of turbulent energy which can lead to reduction of turbulence and possibly flow laminarization in this region. For the backward-facing step, Chandrasuda \& Bradshaw (1981) also observed the importance of normal stress production compared to shear stress production at close to the wall. From their results, at close to the wall, and at some $X / h$ locations, negative total production can also be inferred. Negative production of turbulent kinetic energy was also observed in an axisymmetric jet (Zaman \& Hussain 1980) and a plane mixing layer (Oster \& Wygnanski 1982) at certain locations. Hussain (1986) discussed the possibility of negative production for round jet flow in the presence of leap-frog motions of two adjacent vortex rings during the pairing process. If this pairing motion takes place repeatedly at a location, then negative time-average production is observed. These subharmonics are presumably due to the pairing motion of the fundamental wave approaching from upstream. Thus, it may be conjectured that the negative production observed here may be due to leap-frog type of motion of the vortices undergoing pairing. Previously, it was noted that the shear layer developing after reattachment has an inner and outer shear layer with separate origins. Negative production taking place close to the wall is associated with the inner shear layer.

It is also interesting to observe that at the $Y / h$ location of maximum shear stress production $(Y / h \simeq 1.0)$ (see figure 11$)$, the normal stress production changes its sign. It may also be pointed out that for a wall jet (Zhou et al 1996) similar behaviour between the normal and shear stresses was observed. When excitation is applied, normal production increases while shear production decreases. This may be because large-scale structures get amplified in the near-field region due to excitation and their participation in the pairing process in the reattachment region results in higher values of normal production. Thus, negative production for the excited case is higher than that for the unexcited case. Shear production for the excited case is lower because the velocity gradient is lower in the excited case due to early reattachment. The maximum total production for the unexcited case can be observed to be higher than that of the excited case at $X / h=$ 9.0. This explain why the Reynolds stresses were earlier observed to be smaller for the excited cases.

The drop in total production at $X / h=9.0$ for the excited case should not be taken as an indication that the excitation is responsible for suppressing the turbulence at all locations. Excitation may be responsible for enhancing turbulence in certain regions and suppressing in other regions. To verify this, the turbulent production terms at $Y / h$ locations corresponding to $U / U_{\max }=0.7$ and 0.9 are plotted for both excited and unexcited cases in figure 12. At $U / U_{\max }=0.7$, in the early part of the shear layer $(X / h 3.2)$, the turbulence production is enhanced by excitation while in the later part of the shear layer the turbulence production is suppressed by excitation. The peak turbulence production is essentially doubled with excitation, and the peak location moves upstream by nearly 2 rib 

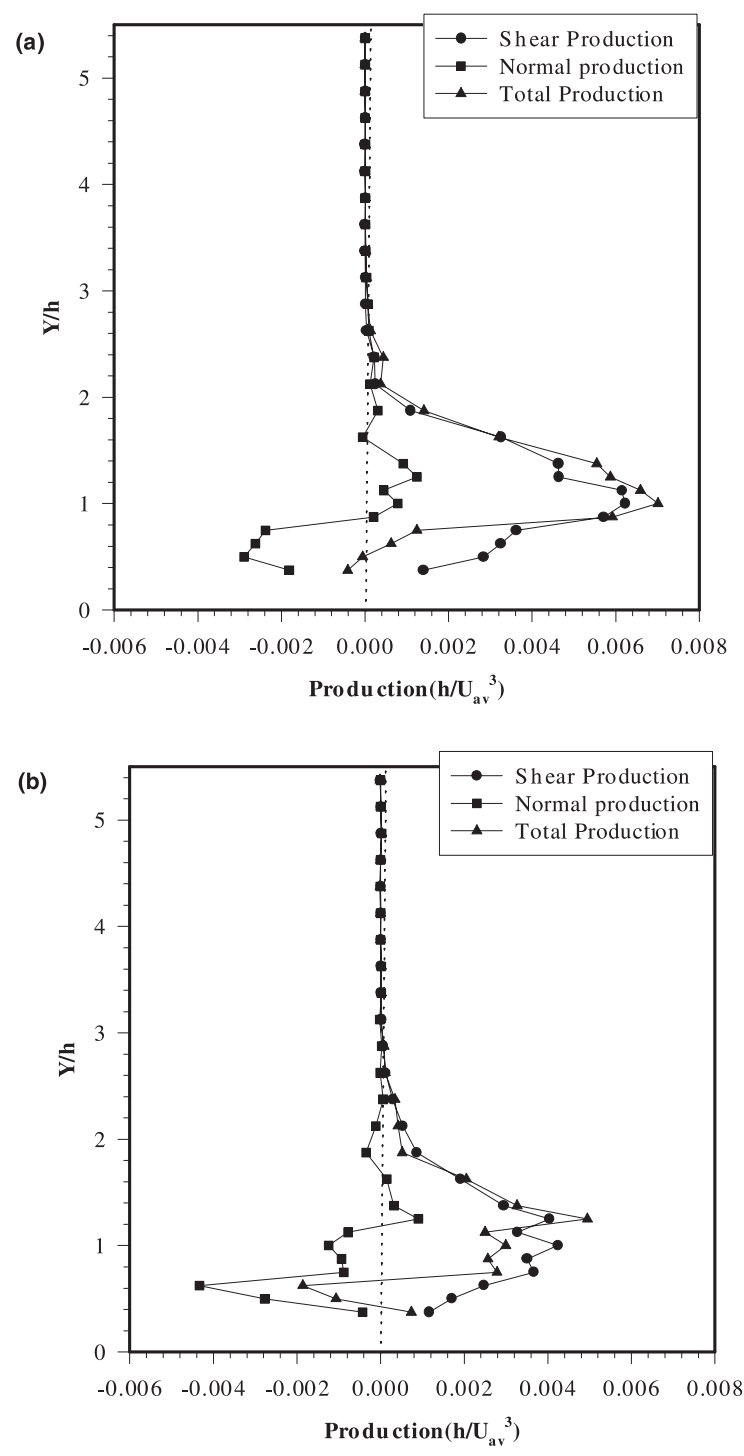

Figure 11. Turbulent energy production at $X / h=9.0$ for the unexcited case (a) and the excited case (b).

heights representing early reattachment. It is supported by the fact that the reattachment point for the natural case is $5.5 h$ and for the excited case is equal to $4.0 h$ indicating $1.5 \mathrm{~h}$ reduction in reattachment length for the excited case. Thus the peak turbulence production location and the reattachment location are possibly correlated with each other. Comparing the production at $U / U_{\max }=0.7$ and $U / U_{\max }=0.9$, it is observed that at $U / U_{\max }=0.9$, the effect of excitation on the turbulence production is not as pronounced. The production for the excited case at $U / U_{\max }=0.9$ is the same order of magnitude as that for the unexcited case. The $U / U_{\max }=0.7$ and 0.9 locations being away from the wall, the normal stress production does not appear to play a big role in the total production.

Dissipation profiles at $X / h=9.0$ for both the excited and unexcited cases are presented in figure 13. For both the excited and unexcited cases, the dissipation is observed to 
(a)
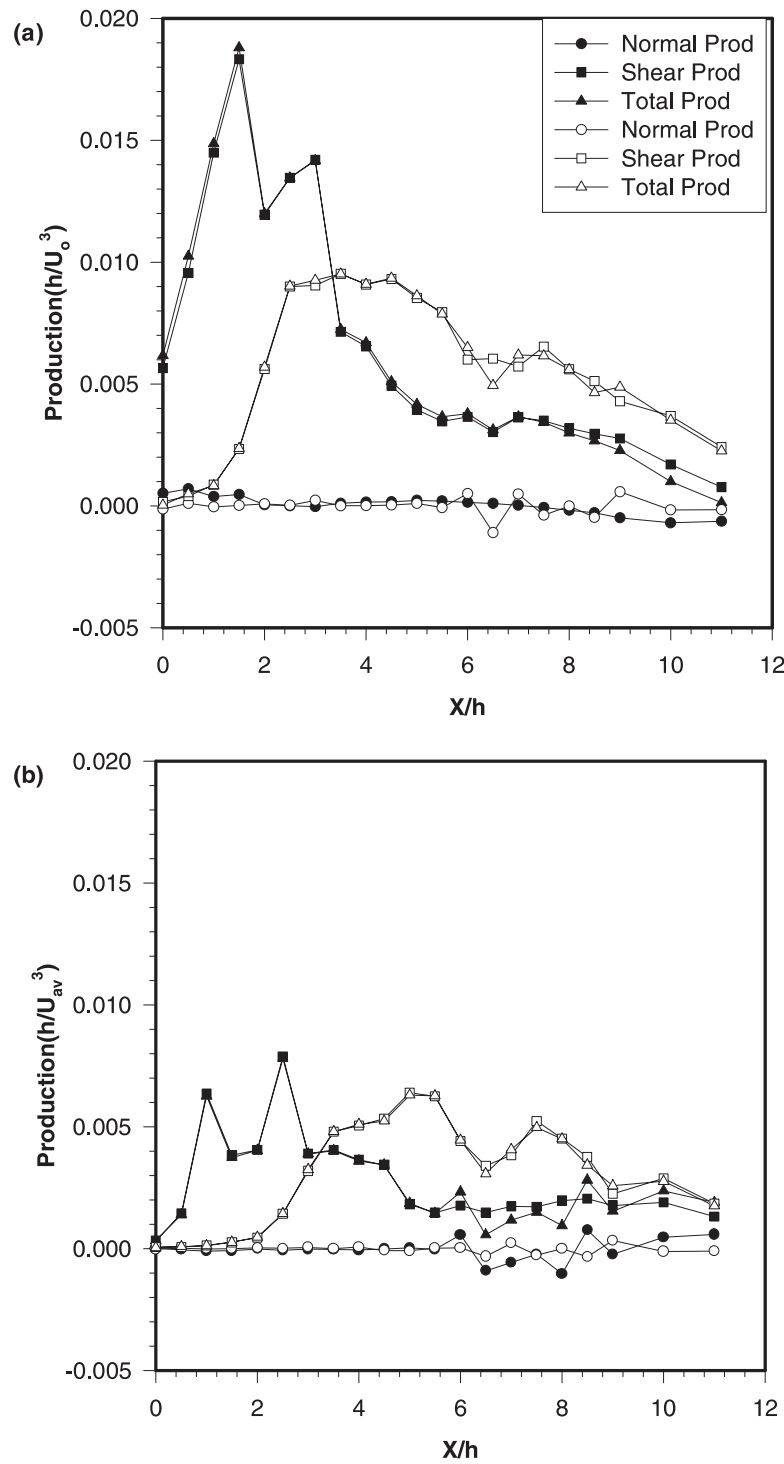

Figure 12. Turbulent energy production at $U / U_{m x}=0.7$ (a) and $U / U_{m x}=$ 0.9 (b) for both excited and unexcited cases.

be maximum at close to the wall and gradually decreases toward the center of the channel. The dissipation is considerably affected by excitation. The dissipation for the unexcited case is observed to be more than that of the excited case close to the wall. This correlates with the near wall production of turbulence (figure 11), where for $Y / h<$ 1 , the production values for the excited case are less than those for the unexcited case. Thus, from equilibrium considerations (production $=$ dissipation), a higher dissipation value may be expected near the wall for the unexcited flow case.

Development of the dissipation both before and after reattachment can be observed at $Y / h$ locations corresponding to $U / U_{\max }=0.7$ and 0.9 in figure 14. Dissipation is higher for the excited flow in the near field region. This is consistent with the observation of Favre-Marinet \& Binder (1979) who imposed a well-controlled large-scale structure on a round jet and took hot wire measurements on the jet axis. Liu (1981) observed 


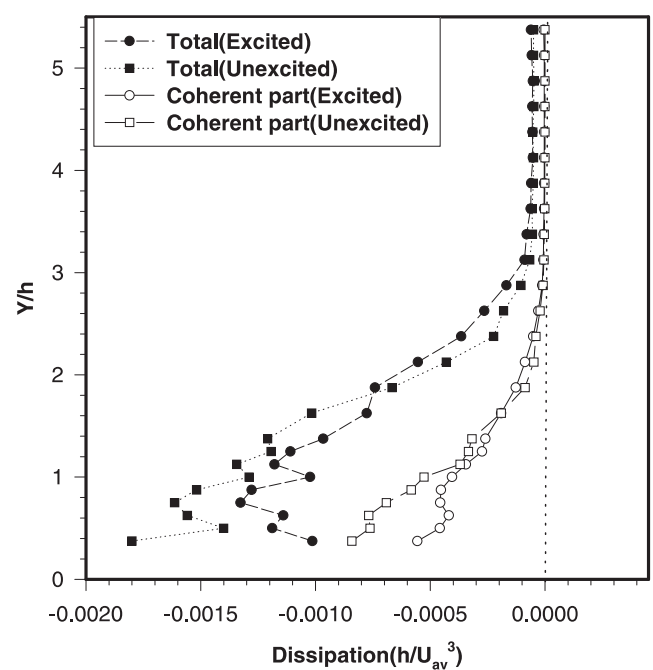

Figure 13. Dissipation profile at $X / h=9.0$ for both unexcited and excited cases.

that fine-grained turbulence occurs at the expense of the large-scale structures and the turbulent dissipation mechanism is stronger for larger initial level of large-scale structures. Both the large-scale production integral and the viscous dissipation integral of smallest eddies had similar variation. Therefore, it may be argued that, in the present case, the dissipation for the excited case is larger than for the unexcited case, due to higher large-scale magnitude. The dissipation is observed to increase in the downstream direction reaching its maximum value at about the reattachment point. It remains constant around the reattachment region and then decreases with subsequent development of the boundary layer. Maximum dissipation occurs earlier for the excitation case which is consistent with earlier flow reattachment. A distinctive feature of the dissipation profile is the cross-over point at about $X / h=8$, beyond which the dissipation values for the unexcited cases exceed those for cases with flow excitation.

From the coherent dissipation contribution at $X / h=9.0$, presented in figure 13 , it is observed that the dissipation is not only due to small-scale eddies but also due to the large-scale eddies as opposed to the traditional belief that the dissipation takes place only due to small-scale eddies. Here, the large-scale eddies are found to be equally responsible for dissipation as the small-scale eddies. This observation supports the argument that the participation of large-scale structures amplified by the external excitation results in higher values of dissipation. It is traditionally believed that large eddies are inviscid. However, it is our belief that dissipation takes place due to the interaction among different large-scale eddies and the interaction between large and small-scales. Hussain (1986) observed that even though coherent structure dynamics is essentially inviscid, there is dissipation within the coherent structures, which can be significant. The results obtained and presented here support the prediction of Hussain (1986) regarding the dissipation mechanism.

The combined energy balance for the unexcited and excited cases at $X / h=9.0$ is shown in figure 15. Pressure transport is observed to play a major role in the turbulent kinetic energy budget for both the excited and unexcited cases. For the unexcited case, the convection and the pressure diffusion are of opposite sign, and further reverse sign at about the same $Y / h$ location $(=1.2)$. Hence, closer to the bottom surface $(Y / h<$ 1.2), most of the turbulent energy influx by pressure diffusion and production is either 

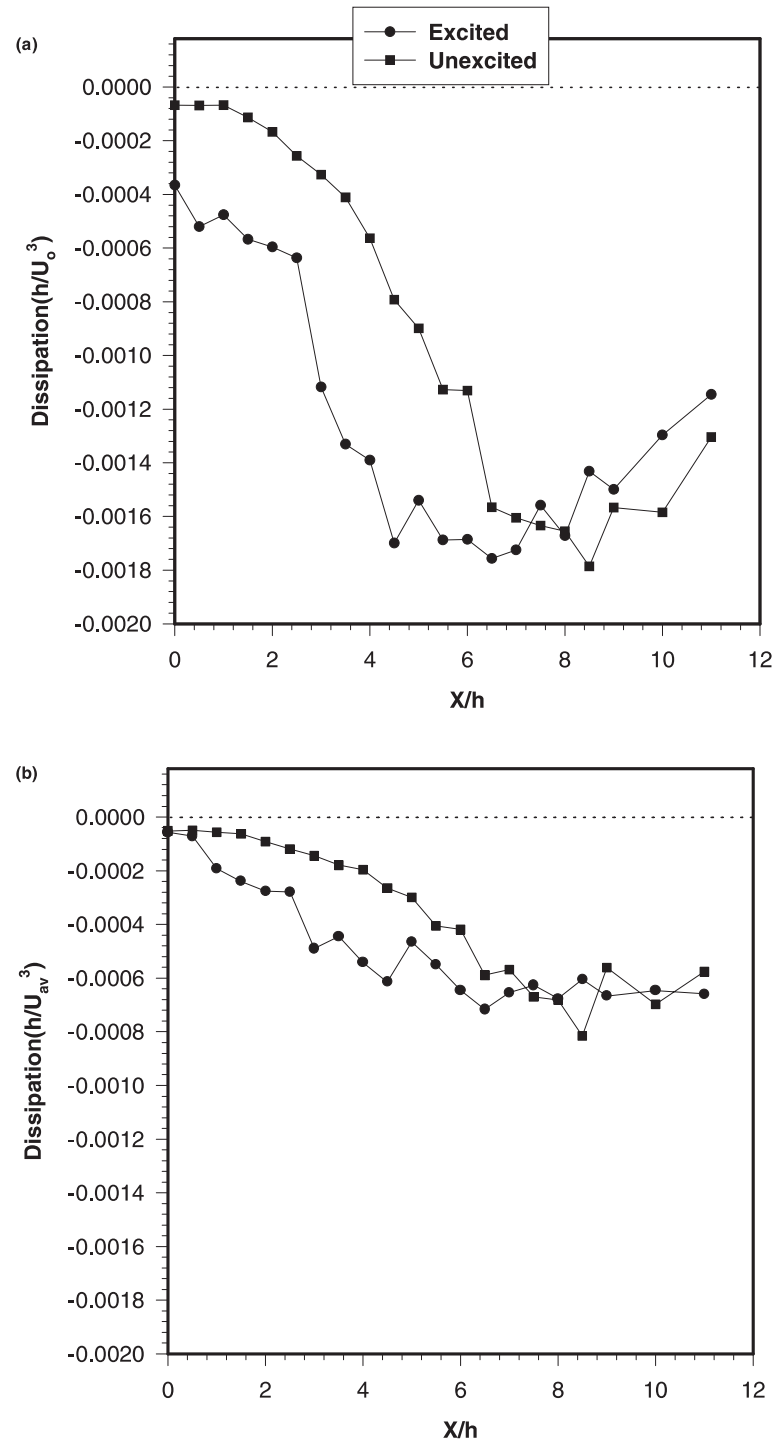

Figure 14. Dissipation profile at $U / U_{m x}=0.7$ (a) and $U / U_{m x}=0.9$ (b) for both unexcited and excited cases.

dissipated or convected away in the downstream direction with the latter mechanism dominating. Away from the wall, the pressure diffusion transport balances the convection, production and diffusion. The pressure diffusion term is observed to be significantly more important than the velocity diffusion. For a wall jet, Zhou et al (1996) observed similar variation in pressure transport, i.e. positive gain in turbulent kinetic energy close to the wall and loss away from it. Driver et al (1982), assumed the pressure diffusion to be negligible and calculated the dissipation by balance. The significance of the pressure diffusion term observed here indicates that its neglect in order to calculate dissipation by balance (Driver et al 1982) may not always be right. For the excited case, at $X / h$ $=9.0$, the pressure transport and convection contribution do not change their sign at any $Y / h$ location. Both convection and pressure transport are observed to be the most dominant turbulent transport quantities. 
(a)

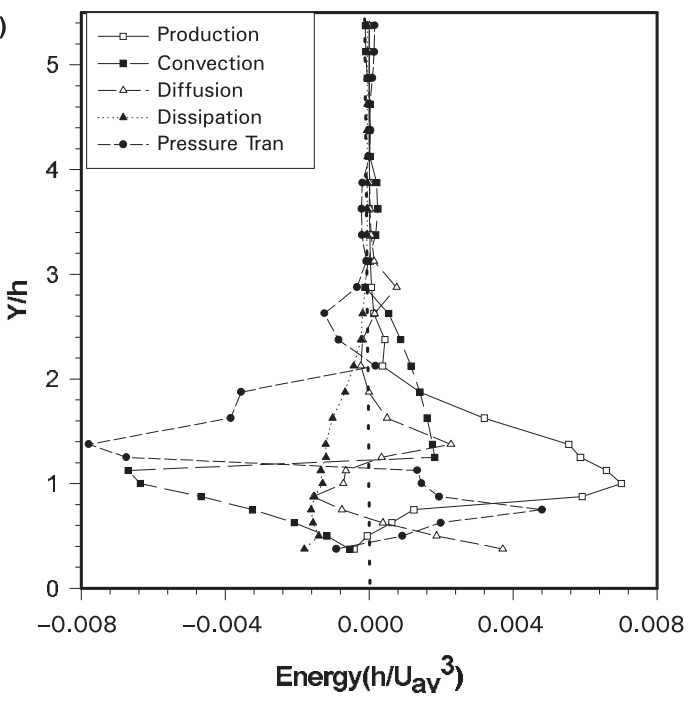

(b)

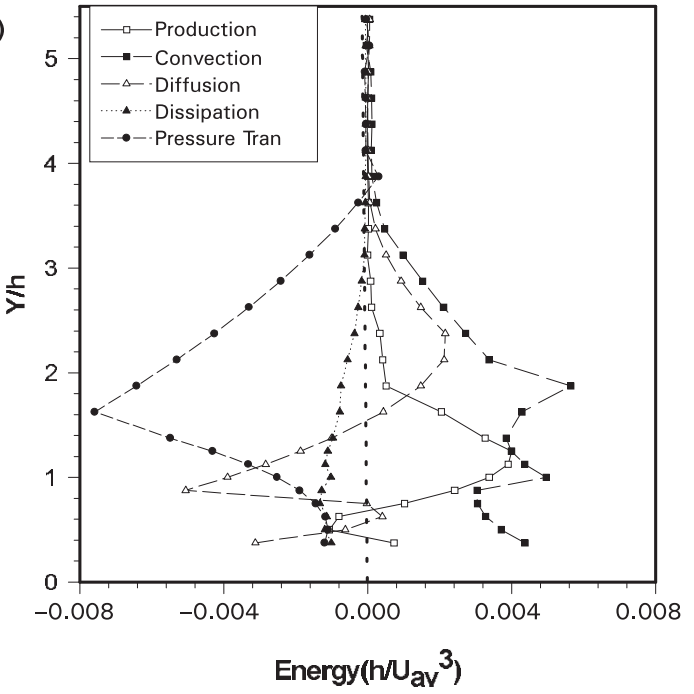

Figure 15. Turbulent kinetic energy budget for the unexcited case (a) and the excited case (b) at $X / h=9.0$.

\subsection{Kinetic energy of coherent eddies}

The coherent energy budget terms at $X / h=9.0$ for both the unexcited and excited cases are presented in figure 16. Comparing the production contribution from the coherent eddies with that of the total production (see figure 15), most of the production is observed to be due to coherent eddies. While comparing the convection contribution for both the cases, the convection contribution is observed to be due to both coherent and non-coherent eddies. Similar observation about coherent convection and production was also observed for wall jet case by Zhou et al (1996). For the unexcited case (figure 16), close to the bottom surface, the coherent eddies are observed to contribute towards the random scale motions (as the intermodal production term is negative), which is be dissipated subsequently. Away from the wall, the intermodal production term is positive indicating 
energy flux from the fine scale to the coherent motion (backscatter). For the excited case (see figure 16), both in the lower and upper part of the shear layer, positive intermodal production is observed. The back scatter observed in the shear layer after reattachment, may be contributing to a renewed organisation of the coherent structures and the presence of large-scale structures well downstream of the reattachment point as observed by Panigrahi \& Acharya (1999) from an octant analysis.

The present results further support the observed backscatter behaviour reported by Knight (1981) and by Hussain (1986). Knight (1981) studied the incompressible temporally developing turbulent mixing layer by imposing a weak perturbation consisting of harmonic and subharmonic disturbance on the initial similarity profiles. For a variety of initial perturbation strengths and wavelengths, energy flux from the fine scale to the coherent
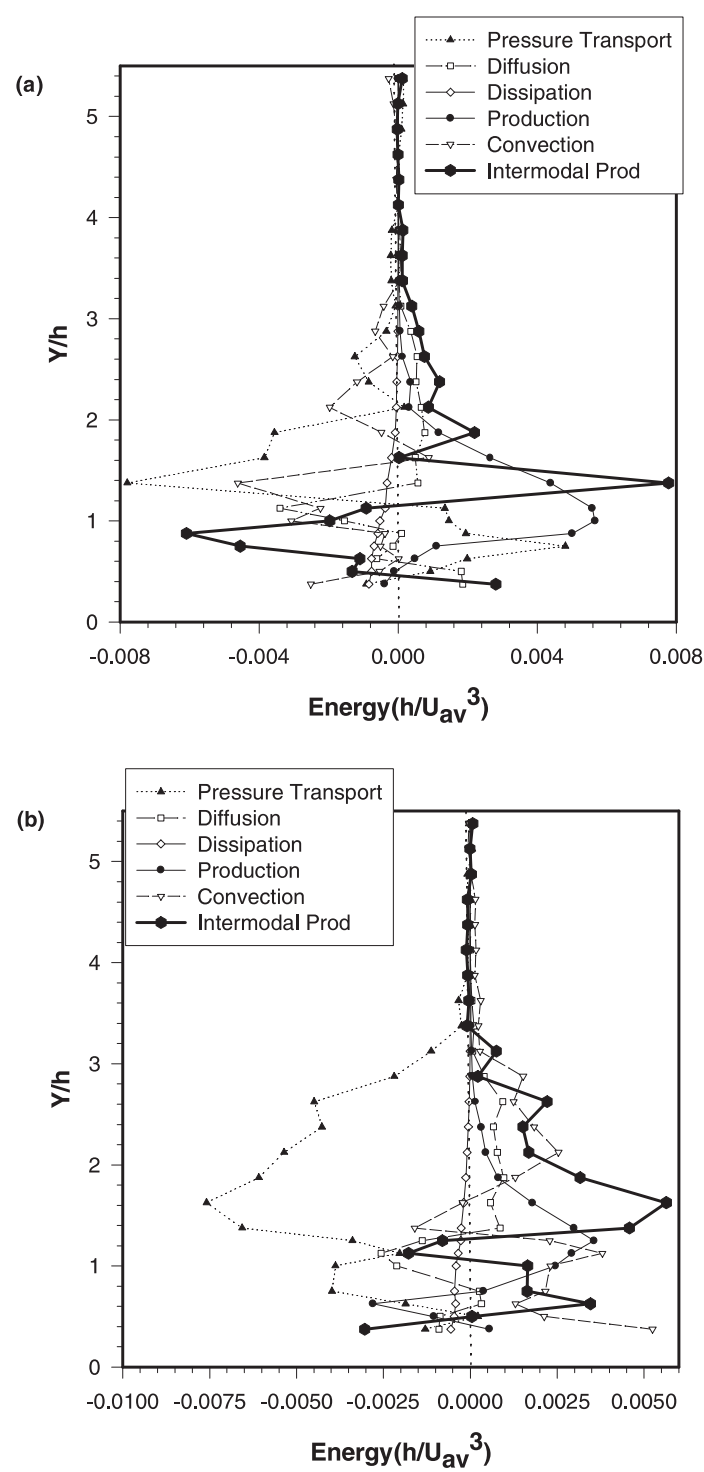

Figure 16. Turbulent kinetic energy budget of the coherent $u$-velocity for the unexcited case (a) and the excited case (b) at $X / h=9.0$. 
scale was predicted during the pairing process. Hussain (1986), in his review paper about coherent structures, observed that the principal contribution to the coherent vorticity comes from the incoherent field. It was shown that the random stretching of random vorticity fluctuations by random velocity fluctuations $\left(w_{r} . u_{r}\right)$ and the random advection of random vorticity by random velocity fluctuations $\left(u_{r} . w_{r}\right)$ can be organised by the coherent structures in such a way as to affect its coherent vorticity.

Note that the intermodal production terms have the largest positive values in the upper part of the shear layers $(Y / h<1.2)$. In this region the velocity is non-Gaussian in nature with higher absolute skewness and flatness values. It was also predicted from quadrant analysis that the large-scale ejection motions were predominant in the upper part of the shear layer. Thus, it seems that in the upper part of the shear layer, the large higher order moments, predominant ejection motions and positive intermodal production are correlated to each other.

\section{Conclusion}

The near-wall behaviour of reattaching flow behind a rib mounted on the surface of a rectangular channel has been studied experimentally for both excited and unexcited cases. The detailed turbulent structures of the reattaching shear layer developing behind the surface-mounted rib are discussed using the mean velocity profiles, the coherent and random stresses, the higher order moments and the turbulent kinetic energy budget. The following observations are made.

(1) The coherent structure magnitude obtained from using the pattern recognition method indicates the significance of large-scale coherent structures in the post reattachment region, contrary to the belief that large scales break into small scales, resulting in predominant presence of small scales after the reattachment region.

(2) The mean velocity profile measurements indicate that $X$-momentum transport is enhanced in the near wall region due to the excitation at the fundamental frequency. The imposed oscillation mostly affects the coherent part of the total fluctuation.

(3) The flow past the reattachment point appears to be characterized by an inner layer and an outer layer. These layers appear to have different origins and retain the initial history well past the reattachment point. Thus the re-developing wall flow is distinctly different from that of a flat plate boundary layer flow. This observation is also supported by the Reynolds stress correlation value that is smaller than that of the flat plate boundary layer.

(4) The higher order moments of the fluctuating $u$-velocity, i.e. skewness and flatness, are observed to depart from the respective Gaussian value of 0 and 3 in the upper part of the shear layer. This departure is attributed to the large-scale intermittent fluctuation, along the outer edge of the shear layer. The quadrant analysis shows that these large-scale motions in the outer edge of the shear layer are predominantly from ejection motions.

(5) The dissipation of turbulent kinetic energy has significant contribution from the coherent structures, contrary to the belief that dissipation is a small-scale phenomena. Rather, it is conjectured that dissipation also takes place due to the interaction between different coherent structures.

(6) The energy budget of the coherent component of turbulent fluctuation shows positive intermodal production in the outer region of the shear layer indicating a reverse cascade mechanism. In the upper shear layer region, the higher order moments, the 
large-scale ejection motions and the backscatter phenomena seem to be correlated with one another.

\section{List of symbols}

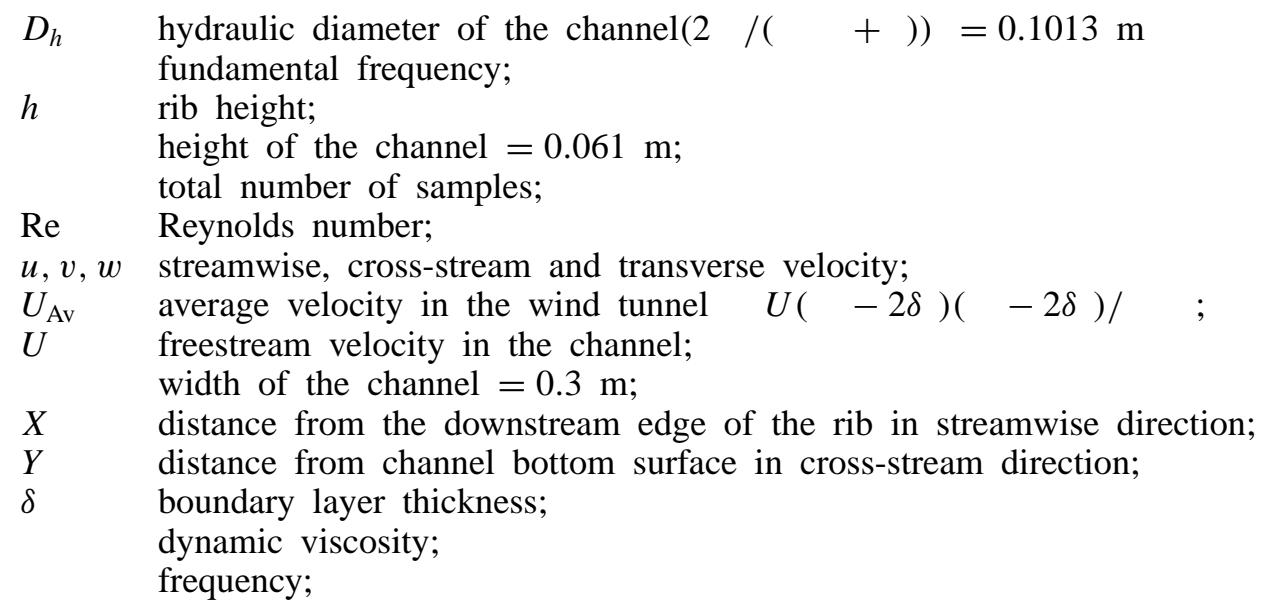

\section{Subscripts}

c coherent;

$r$ random;

fundamental component;

$s \quad$ subharmonic component;

max maximum;

tot total.

\section{References}

Bhattacharjee S, Scheelke B, Troutt T R 1986 Modification of vortex interactions in a reattaching separated flow. AIAA J. 24: 623-629

Champagne F H 1978 The fine scale structure of the turbulent velocity field. J. Fluid Mech. 86: $67-108$

Chandrasuda C, Bradshaw P 1981 Turbulence structure of a reattaching mixing layer. $J$. Fluid Mech. 110: 171-194

Driver D M, Seegmiller H L 1982 Features of a reattaching turbulent shear layer subject to an adverse pressure gradient. AIAA/ASME 3rd Joint Thermophysics, Fluids, Plasma and Heat Transfer Conference, St Louis, Missouri, AIAA-82-1029

Favre-Marinet M, Binder G 1979 Structur des jets pulsants. J. Mech. 18: 356-394

Hasan M A Z 1992 The flow over a backward facing step under controlled perturbation: Laminar separation. J. Fluid Mech. 238: 73-96

Hinze J O 1975 Turbulence 2nd edn (New York: McGraw-Hill)

Ho C M, Huere P 1984 Perturbed free shear layers. Annu. Rev. Fluid Mech. 16: 365-424

Hussain A K M F 1970 The mechanics of a perturbation wave in turbulent shear flow. Dissertation, Stanford University

Hussain A K M F 1986 Coherent structures and turbulence. J. Fluid Mech. 173: 303-356 
Hussain A K M F, Zaman K B M Q 1980 Vortex pairing in a circular jet under controlled excitation. J. Fluid Mech. 101: 493-544

Knight D D 1981 Numerical investigation of large-scale structures in the turbulent mixing layer. Symposium on Turbulence Proceedings of the Sixth Symposium on Turbulence in Liquids, Department of Chemical Engineering, University of Missouri-Rolla, pp 167-177

Kundu P K 1990 Fluid mechanics (New York: Academic Press)

Liu J T C 1981 Interaction between large-scale coherent structures and fine-grained turbulence in free shear flows. Transition Turbulence: 167-214

Lumley J L 1981 Coherent structures in turbulence. Transition Turbulence: 215-242

Oster D, Wygnanski I 1982 The forced mixing layer between parallel streams. J. Fluid Mech. 123: 91-130

Panigrahi P K 2001 Fundamentally excited flow past a surface-mounted rib. Part I: Turbulent structures characterization. Sadhana (this issue)

Panigrahi P K, Acharya S 1999 Mechanisms of turbulence transport in a turbine blade coolant passage with a rib turbulator. J. Turbomachinery 121: 152

Roos F W, Kagelman J T 1986 Control of coherent structures in reattaching laminar and turbulent shear layers. AIAA J. 24: 1956-1963

Zaman K B M Q, Hussain K M F 1980 Vortex pairing in a circular jet under controlled excitation Part I. General jet response. J. Fluid Mech. 101: 449-491

Zhou M D, Heine C, Wygnanski I 1996 The effects of excitation on the coherent and random motion in a plane wall jet. J. Fluid Mech. 310: 1-37 